\title{
Experiencia colaborativa de la Mesa Técnica contra la Explotación Sexual Comercial de la niñez en Bíobio
}

\author{
Verónica Patricia Gómez Fernández ${ }^{1,}$ \\ Fania Leticia Cid Arias ${ }^{2}$
}

\begin{abstract}
RESUMEN
La sistematización al trabajo de la Mesa Técnica Regional Contra la Explotación Sexual Comercial de Niños, Niñas y Adolescentes (ESCNNA) de la Región del Biobío de Chile, da cuenta de los esfuerzos de esta instancia por sensibilizar y movilizar a garantes de derechos para la coconstrucción de un entorno de protección integral para niños, niñas y adolescentes en riesgo, y un soporte social en aquellos ya víctimas, articulando diversos actores, desde una perspectiva colaborativa de responsabilidad y justicia social.

Se reconstruye y reflexiona sobre cinco años de trabajo en el complejo abordaje de una problemática de extrema vulneración y con múltiples manifestaciones, cuya base son factores estructurales que naturalizan la violencia sexual y el daño causado a las víctimas, siendo la explotación sexual, finalmente, el resultado de una cadena de traumas y abandonos.

Este artículo es una producción colectiva de conocimiento intencionado para transformar la práctica social en el tema.
\end{abstract}

Palabras clave: sistematización, ESCNNA, mesa técnica, garantes de derechos

\section{Collaborative experience at the Technical Board to Combat Commercial Sexual Exploitation of Children in Bíobio} SUMMARY

Systematization of the work performed by the Regional Technical Board to Combat Commercial Sexual Exploitation of Children and Young People (ESCNNA) in Chile's Biobío Region reveals the efforts made by this body to build awareness and mobilize guarantors of rights to jointly build an environment of integrated protection for children and young people at risk, together with social support for those who have already fallen victim,

Chilena. Trabajadora Social, Académica Departamento Ciencias Sociales, Universidad Católica de la Santísima Concepción. E-mail: vegomez@ucsc.cl

2 Chilena. Licenciada en Trabajo Social Universidad Católica de la Santísima Concepción. E-mail: fcid@tsocial.ucsc.cl 
Experiencia colaborativa de la Mesa Técnica contra la Explotación Sexual Comercial de la niñez en Bíobio / Gómez y Cid

drawing together diverse stakeholders under a collaborative perspective of social justice and responsibility.

This work reconstructs and discusses five years of work in complex task of addressing a problem of severe breaches of rights in multiple manifestations, based on structural factors that naturalize sexual violence and the harm caused to victims, with sexual exploitation arising as the end result of a chain of traumas and abandonments.

This article is a collective work of knowledge designed to transform social practices in this field.

Keywords: systemization, ESCNNA, technical board, rights guarantors

\title{
Experiência colaborativa da Mesa Técnica contra a Exploração Sexual Comercial da infância no Bíobio
}

\begin{abstract}
A sistematização do trabalho da Mesa Técnica Regional Contra a Exploração Sexual Comercial de Crianças e Adolescentes (ESCNNA) da Região do Biobío do Chile, apresenta os esforços desta instância por sensibilizar e mobilizar a garantidores de direitos para a co-construção de um entorno de proteção integral para crianças e adolescentes em risco, e um suporte social para aqueles já vítimas, articulando diversos atores, desde uma perspectiva colaborativa de responsabilidade e justiça social.

São reconstruídos e é feita uma reflexão sobre cinco anos de trabalho na complexa abordagem de uma problemática de extrema vulneração e com múltiplas manifestações, cuja base são fatores estruturais que naturalizam a violência sexual e o dano causado às vítimas, sendo a exploração sexual, finalmente, o resultado de uma cadeia de traumas e abandonos.

Este artigo é uma produção coletiva de conhecimento intencionado para transformar a prática social no tema.
\end{abstract}

RESUMO

Palavras-chave: sistematização, ESCNNA, mesa técnica, garantidores de direitos

\section{Introducción}

La sistematización de experiencias es un proceso de reflexión e interpretación crítica sobre la práctica y desde la práctica, que se realiza con base en la reconstrucción y ordenamiento de los factores objetivos y subjetivos que han intervenido en esa experiencia, para extraer aprendizajes y compartirlos (Jara, 2012). Se conside- 
ra como un modo de gestión de conocimiento que se construye en las experiencias (Ghiso, 2008). Por otro lado, es una práctica con sello latinoamericano, que posibilita buscar respuestas para reflexionar, intervenir ética, idónea y responsablemente, y transformar nuestras realidades (Cifuentes, 1999). Además, busca reconocer, desde la voz de sus protagonistas, las lógicas y las construcciones de sentidos de las prácticas sociales. Se trata de una producción de conocimiento "desde abajo" (Fundación Renacer, 2011, p. 12). Este proceso permite impulsar el aprendizaje crítico de forma intencionada y vinculando el pensamiento, las emociones y las acciones que permitan ampliar el bagaje teórico actual desde la comprensión del dinamismo y la globalización, por el cual la Mesa Técnica logra anteponerse y levantar la riqueza de su trayectoria como aprendizaje para mejorar sus procesos de intervención en explotación sexual comercial de niños, niñas y adolescentes (desde ahora en adelante ESCNNA) en beneficio directo de los niños, niñas y adolescentes.

De acuerdo con lo anterior, la Mesa Técnica contra la ESCNNA se apropia críticamente de los saberes y sentires en la reflexión de sus acontecimientos, que puedan contribuir como guía para la acción trasformadora desde sus propias protagonistas, constituyendo la intervención profesional el foco principal de la experiencia sistematizada.

El trabajo de sistematización se abordó desde las dimensiones metodológica, epistemológica y ontológica, y se realizó sobre la base de un trascurso activo de intervención que busca reflexionar y comprender lo ya realizado, integrando los elementos contingentes en los cuales las participantes se encuentran desarrollando su acción.

\section{Antecedentes y contextualización}

A continuación se presentan elementos que facilitan la comprensión en temática de ESCNNA en diferentes aspectos territoriales, demográficos, sociales, institucionales y programáticos, en los cuales la Mesa Técnica se desenvuelve. 
La población de la región del Biobío, según CENSO 2017, alcanza a 2.037.414 personas. De las cuales 382.035 corresponden a menores de 18 años. Un 18,9\% de la población total se encuentra en condición de pobreza multidimensional ${ }^{3}$, pese a que la región es una de las principales concentradoras de importantes actividades económicas del país, de rubros tan diversos como la siderurgia, la agricultura tradicional, la industria de la celulosa, la actividad forestal, la generación de electricidad, etc.

Se estima que al menos 3.719 niños, niñas y adolescentes son víctimas de explotación sexual comercial en Chile. No obstante, la experiencia de trabajo con las víctimas lleva a plantear la necesidad de considerar que muchas de las formas de ESCNNA son poco visibles y ameritan profundizar a través de otros estudios (ONG Raíces, s/f, p.5).

La explotación sexual comercial, como tal, no está definida jurídicamente, siendo sancionada mediante procedimientos penales relacionados con la "obtención de servicios sexuales y facilitación de la prostitución", produciendo una deficiencia en el sistema judicial para la formulación del delito, producto de la falta en la tipificación penal y ausencia del término "explotación sexual".

La figura de ESCNNA se sanciona desde protocolos, como la Ley 20.507 contra la trata; Ley 19.927, referida a la "prostitución infantil” en el artículo 367; y el artículo 367 quáter, sobre la obtención de servicio sexuales. Por lo tanto, al no poseer una definición jurídica, se penaliza conductas asociadas desde el Código Penal, el Código de Procedimientos Penales y el Código Procesal Penal en materia de delitos de pornografía infantil. Esta última considera la cadena de producción, comercialización, obtención y almacenamiento; sin embargo, en el ámbito de turismo no se cuenta con soporte legal específico o relacionado. Barra (2014) señala que son significativos, aunque no suficientes, los avances que ha logrado el

\footnotetext{
Informe de Desarrollo Social 2018. Recuperado de http://www. ministeriodesarrollosocial.gob.cl/storage/docs/Informe de Desarrollo $\underline{\text { Social 2018.pdf }}$
} 
Estado chileno en materia de protección a niñas, niños y adolescentes que han sido víctimas de abuso grave, entre ellas explotación sexual comercia (ESC).

A su vez, en cuanto a la legislación y el acceso a la justicia en ámbitos de protección a las víctimas, el sistema presenta varias deficiencias, tales como la permanencia del testimonio de la víctima, al no poder prescindir de ella en el proceso; un exceso de entrevistas y peritajes, provocando una victimización secundaria; escasez de recursos para la protección y seguridad en la integridad de la víctima, unido a la desconfianza en el proceso, provocando una retirada de la denuncia, por lo cual el caso no continua su proceso, generando sensación de impunidad.

El informe de monitoreo del país sobre la ESCNNA (ECPAT, 2014) identifica una serie de impedimentos en materia legislativa, mencionando una gran dificultad que tiene relación con el término "obtención de servicios sexuales", pues implica que el intercambio sexual tiene que haber sido realizado para poder sancionar el requerimiento de servicios sexuales. Si la acción penalizada fuera de requerimiento, sería más fácil interceder ante el intento de un cliente de hotel de llevar a un o una menor de 18 años a su habitación (ECPAT, 2014).

Por falta de una Ley de Protección integral para niñas, niños y adolescentes, y la falta en la tipificación de la ESCNNA, no existen estadísticas que den cuenta de la cantidad de causas registradas referentes a la explotación sexual comercial y, asimismo, en ocasiones el delito es configurado como abuso o violación. Sin embargo, el Informe de Monitoreo del País sobre la ESCNNA hace mención de una publicación del diario La Tercera sobre 479 investigaciones iniciadas bajo esa figura penal entre 2007 y 2011, según cifras entregadas por Fiscalía Nacional. Si se compara esta cifra con los 25.416 casos de delitos sexuales registrados durante 2013, en $74 \%$ de los cuales las víctimas eran menores de 18 años y aun considerando el subregistro, es evidente la baja judicialización de la explotación sexual comercial de Chile. Del total de delitos sexuales 
Experiencia colaborativa de la Mesa Técnica contra la Explotación Sexual Comercial de la niñez en Bíobio / Gómez y Cid

mencionados el $63,5 \%$ obtuvo salidas no judiciales y solo el 9,87\% obtuvo sentencia definitiva condenatoria (ECPAT, 2014).

La estrategia pública de intervención en ESCNNA está transitando un camino que busca involucrar cada vez a más al Estado, actores y organizaciones en desnaturalizar esta práctica, que reduce a los seres humanos NNA a la calidad de mercancías e incide, de manera brutal y dañina, en su desarrollo físico, emocional e intelectual (Araya, Retuerto y Tarducci, 2012).

Los ingresos a los programas del departamento de Protección de Derechos de SENAME se concentran en la categoría víctima de abuso sexual y maltrato, representada por un total de 43.292 ingresos en todo el país, siendo la región del Biobío 2.166 NNA de un total de 6.835 (SENAME, 2016).

Los ingresos al área de protección judicial en tribunales de familia por causal de explotación sexual corresponden a seis causas con igual número de víctimas. La explotación con fines comerciales registra preeminencia absoluta, con un $78,3 \%$; a distancia se ubican otras dos causales con frecuencias superiores a 5,0\%, como la utilización de niños, niñas y adolescentes en actividades sexuales remuneradas, que ascienden a 14\%, así como la utilización de niños, niñas y adolescentes para producción, promulgación y divulgación de pornografía, con un 5,7\% del total. De este modo, casi la totalidad de los ingresos corresponden a NNA cuya problemática común se asocia a actividades con presencia organizada en el ámbito del comercio sexual (SENAME, 2016).

Los proyectos especializados en la atención de niños, niñas y adolescentes víctimas de explotación sexual comercial (PEE) son ejecutados por el Servicio Nacional de Menores (SENAME), en convenio con organismos colaboradores acreditados. En la Región del Biobío, PEE AURA, de Fundación Novo Milenio, es el único proyecto ambulatorio destinado a la acogida y restitución de derechos de los NNA, con cobertura de 75 niñas y niños entre 10 a 18 años (SENAME, 2017). 
La Mesa Técnica Regional nace en el 2012 como resultado del proyecto "Iniciativas Regionales contra la Explotación Sexual de Niños, Niñas y Adolescentes" en regiones del país, participando instituciones públicas, privadas y organizaciones de la sociedad civil, con directa relación en el trabajo de niños, niñas y adolescentes, para la generación de propuestas en políticas públicas contra la ESCNNA acordes a su contexto y territorio.

La Mesa Técnica posee un enfoque regional, abarcando las provincias de Biobío, Ñuble, Concepción, y Arauco, conformada por diferentes representantes de organizaciones claves en la temática y considerando para el cumplimento de sus objetivos, sus cualidades y diferenciaciones dentro del territorio.

Por medio del proyecto base, se logra consolidar una Mesa Técnica Contra la ESCNNA, siendo la única Región que ha logrado perdurar al término del proyecto, administrada en sus inicios por SENAME; actualmente se establece una coordinación rotativa, asumiendo desde 2017 la Universidad Católica de la Santísima Concepción su representatividad.

Como objetivo general, señala: "Propender a desnaturalizar la Explotación Sexual Comercial de Niños, Niñas y Adolescentes (ESCNNA), considerada como una forma moderna de esclavitud y una práctica cruel de abuso de poder"4.

La Mesa Técnica es conformada por distintas instituciones del Estado y privadas, con un equipo de trabajo multidisciplinario voluntario, en su mayoría de profesionales de las ciencias sociales, los cuales han visibilizado con diversas voces y en distintos espacios la temática, en el afán de favorecer la desnaturalización de la ESCNNA y su visibilización, mediante acciones de sensibilización y difusión destinadas a aquellos actores garantes y cogarantes de derechos de los NNA. Entre sus discursos destaca: "La explotación sexual comercial de niños, niñas y adolescentes: Una responsa-

Fuente: Plan de acción Mesa Técnica contra la ESCNNA, Región del Bío Bio, 2012. 
bilidad de todas y todos". Afirman que la única manera de intervenir esta complejidad ha sido siempre sumando cada vez más personas, organizaciones, medios de comunicación, universidades, de manera de contribuir a fortalecer cada vez más la red de protección formal e informal hacia los niños, niñas y adolescentes más vulnerados (Araya, Retuerto y Tarducci, 2012, p. 13). También significó asumir un trabajo sistemático, para sensibilizar y visualizar la problemática en distintas instituciones y sus causas estructurales, culturales, políticas, económicas y sociales; potenciadas por la indiferencia y negligencia del mundo adulto, la falta de amor que termina en dependencias y focos de daños con explotadores que en ocasiones los NNA víctimas defenderán, ya que se han convertido en su único refugio de "protección". No se puede actuar solo frente a este gran monstruo, se necesitan múltiples ojos y oídos para detectar situaciones de riesgo (Araya, Retuerto y Tarducci, 2012, p.8). Sin embargo, lograr que una comunidad incida en las prácticas de intervención contra ESCNNA depende de muchos factores, se necesita comprometer diálogos permanentes, participación, movilizar y aceptar la diversidad de estrategias, en las que cada uno podrá ser parte de un entorno de protección efectivo para los NNA.

Con la finalidad de realizar un acercamiento a los significados de las víctimas, los procesos de intervención en los que participaron y sus contextos inmediatos, las integrantes de la mesa, con el apoyo de la Universidad Católica de la Santísima Concepción, ejecutaron un proyecto de investigación de tipo cualitativo y de carácter fenomenológico entre los años 2015-2017, denominado: "Trayectorias, sentidos y sentimientos: una reconstrucción desde quienes fueron NNA víctimas de ESC”, que profundiza en la postintervención del proyecto de la Fundación Novo Milenio y el Servicio Nacional de Menores. El objetivo general que se planteó fue: rescatar las vivencias de los y las que fueron niños y niñas víctimas de explotación sexual y participantes del Proyecto Aura, a través de la reconstrucción de sus trayectorias de vida. Lo anterior se especifica en los siguientes alcances: Identificar 
hitos, mitos, sucesos y vivencias reconocidas como fundamentales en la reconstrucción de las biografías de los participantes; identificar los sentidos y significados que los protagonistas les otorgan a todas aquellas personas y acciones que fueron parte de su vida; reconstruir los contextos socioestructurales y discursivos implicados que rodean los sucesos de los protagonistas; determinar los discursos dominantes y fundamentos paradigmáticos de base de los procesos de intervención en los que se vieron envueltos los protagonistas; favorecer climas de confianza, seguridad y participación para la construcción de las trayectorias de vida y el uso posterior de la información; revisar las condiciones de la reconstrucción de los relatos, por parte de las circunstancias y los sujetos; visibilizar y problematizar la ESCNNA desde un enfoque de derechos ${ }^{5}$.

\section{Marco metodológico del proceso de sistematización}

El plan de trabajo de la sistematización incluyó las siguientes etapas: diseño del proyecto de sistematización; el trabajo de campo, que incorporó la reconstrucción e interpretación; la socialización de los resultados con la Mesa Técnica, y la elaboración del informe final cuyo resumen da origen a este artículo.

La población que participó del proceso de sistematización corresponde a integrantes de la Mesa Técnica por un periodo superior a dos años $(\mathrm{P})$ :

P1: Representante de Fiscalía Regional del Biobío del Ministerio Público, de profesión abogada.

P2: Directora Proyecto de Reparación del Daño de Víctimas de ESCNNA Aura Coronel al Sur, Fundación Novo Milenio, de profesión profesora de Educación General Básica.

Fuente: Proyecto DIN / 2015 Facultad Comunicación, Historia y ciencias Sociales, UCSC. 
Experiencia colaborativa de la Mesa Técnica contra la Explotación Sexual Comercial de la niñez en Bíobio / Gómez y Cid

P3: Directora Proyecto de Reparación del Daño de Víctimas de ESCNNA Aura Concepción, Fundación Novo Milenio de profesión profesora de Educación General Básica.

P4: Representante del Servicio Regional del Ministerio de Educación, de profesión educadora diferencial.

P5: Representante del Servicio Regional de Turismo, de profesión arquitecta.

P6: Académica de la Universidad Católica de la Santísima Concepción, doctora en Antropología.

P7: Académica de la Universidad Católica de la Santísima Concepción, de profesión trabajadora social, magíster en Ciencias de la Familia.

El foco fundamental de esta sistematización fue la experiencia en la intervención social de la Mesa, conscientes de que los conocimientos que se producen en la práctica son diferentes a los que se logran a través de la investigación (Barnechea \& Morgan, 2010).

Se entiende la intervención social de la Mesa en ESCNNA como la integración del qué y el cómo. El qué corresponde al objeto y el cómo al proceso (Cifuentes, 2015). Se analizó el modelo de trabajo (eje metodológico), reflexionando sobre sus conocimientos (eje ontológico) y procesos de conceptualización, tanto de su trabajo como de los paradigmas utilizados (eje epistemológico). Se posicionó desde un paradigma constructivista, que permitió las narrativas de las participantes directas y sus experiencias a través del relato cualitativo y trasformador. Se asumió que la relación entre objeto-sujeto es la construcción de interacciones sociales, con un aprendizaje activo, dinámico y trasformador. Se utilizó un método asociado al interaccionismo simbólico, para derivar sistemáticamente teorías sobre el comportamiento humano y el mundo social en una base empírica (Jiménez, García, Azcárate, Navarrete y Cardeñoso, 2016). 
El constructivismo establece que aquello que conocemos y creemos resulta del lenguaje con el que entendemos y transmitimos nuestras percepciones del mundo. Al hablar - en un mundo compartido siempre con otros - creamos la realidad. Por eso, un mismo fenómeno, una misma realidad, puede ser construida de diferentes formas en función de distintos puntos de vista (Lindón, 2007).

La perspectiva desarrollada asume que las acciones sociales que produjeron las participantes fueron determinadas según su forma de percibir, entender, interpretar, juzgar y organizar su mundo. Para investigar las acciones sociales, la vía más propicia fue preguntar directamente a las participantes por qué actúan de determinada manera y observarlas en sus interacciones cotidianas. Esto permitió comprender cómo y por qué actúan tal como lo hacen, y a qué significados y sentidos obedecen sus acciones (Pérez, 2005).

Se integró al marco de referencia de la sistematización la teoría de la complejidad (Morin, 2011), la cual define el conocimiento intelectual o la comprensión de la realidad desde un pensamiento complejo, desplazando la simplicidad y reduccionismo como respuesta del sujeto frente a su realidad; esta forma de sistematización comprende que el conocimiento individual es ambiguo y desordenado, y requiere ser pulido por medio de la retroalimentación de manera multidisciplinaria y multirreferencial, desde un análisis profundo con elementos relacionados con su entorno, desarrollando el principio de la Holo gramática: ver las partes del todo y el todo de las partes. La amalgama de interacciones de un sistema es tal que no pueden concebirse solo analíticamente, por lo que no tiene sentido proceder vía el conocimiento de variables aisladas para dar cuenta de un conjunto o subsistema complejo (Luengo, 2008). Vivimos bajo el imperio de los principios de disyunción, reducción y abstracción, cuyo conjunto constituye el “paradigma de simplificación” (Morin, 2011).

Esta sistematización no buscó establecer un saber único, sino más bien reconocer la riqueza de la diversidad, el intercambio cons- 
tante de los saberes que logra producir un conocimiento atingente a la necesidad de un mundo globalizado, con cambios estructurales y, más bien, desde una problemática tan compleja como la ESCNNA.

Como estrategia de análisis, la teoría fundamentada permitió la identificación de procesos sociales mediante la construcción de teorías a partir de los datos, permitiendo una vinculación constante de la realidad cotidiana en la que surge y el marco teórico utilizado. Lo anterior posee directa vinculación con el objetivo de esta sistematización: el reconocimiento del hecho social e interpretación de datos desde su contexto y relación con las participantes, como fuente de conocimiento que permite generar teorías, que logre describir, explicar y comprender "desde el interior" hacia el mundo de "ahí afuera" (Kvale, 2011), con la capacidad de crear conocimientos desde la experiencia y sus interpretaciones para unir la teoría como apoyo, más que fundamento inmovible.

Este proceso se llevó a cabo por medio de tres etapas, momentos de reconstrucción histórica, de memoria narrada. Esto remite a recuerdos personales, con perspectiva singular y general, que se interrelacionan y aportan visión general y específica (Londoño \& Atehortúa, 2011).

1. Diseño: momento de búsqueda de apoyo en referentes teóricos, otras sistematizaciones, selección estratégica de procesos de investigación, todo lo necesario para concretar el plan de trabajo.

2. Reconstrucción y análisis (abierta/axial/selectiva): modelo intermedio, por el cual se comenzaron a integrar datos teóricos y empíricos, mediante la codificación de la información.

3. Teorización y generación de conocimiento: fase de aproximación a los análisis fundamentados e identificación de nuevos hallazgos para la generación de nuevas propuestas. Significó el esfuerzo de construir relaciones entre la teoría y la práctica o de construcción de teoría a partir de los datos. 
El estudio tuvo como objetivo general de sistematización: "Reconstruir el proceso de trabajo de la Mesa Técnica Contra la ESCNNA región del Biobío, en sus aspectos metodológicos, trayectoria del proceso y conocimiento obtenido, desde las experiencias reflexivas de sus participantes y lógicas colaborativas tendientes a mejorar las estrategias de intervención".

Los objetivos específicos fueron:

1. "Reconocer metodología de trabajo de la Mesa Técnica Regional Biobío contra ESCNNA, utilizada durante su trayectoria”.

2. "Analizar el desarrollo del proceso en el trabajo de la Mesa Técnica Regional Biobío contra ESCNNA, a través de la identificación de facilitadores y nudos críticos".

3. "Develar los aprendizajes de la práctica social intersectorial de la Mesa Técnica con intencionalidad de transformación".

Las técnicas implementadas fueron: revisión documental de registros, informes, evaluaciones, etc. Talleres grupales con utilización de las técnicas "El Camino", "Flujograma", grupos focales y entrevistas semiestructuradas, además de la observación participante en distintas reuniones y actividades de la Mesa. La información se resguardó por medio de un registro de transcripciones "en vivo" de cada participante, textual y cronológicamente. Para ordenar este registro se generó una matriz de reconstrucción que ayudó a la visualización temática y a producir las categorías.

\section{Análisis y discusión}

Una vez reconstruida la historia consensuada, utilizando una lógica de comprensión según lo expresado por las participantes y desde la fidelidad en la trascripción sobre sus relatos, se identifican los principales hallazgos por medio de las reflexiones des-

Barudy describe esta técnica como autobiográfica en su manual de técnicas para promover la resiliencia en niños, niñas y adolescentes. 
de los ejes temáticos. Se reflexiona la información ordenada para afianzar la configuración de sentidos y visiones y comprensiones particulares situadas (Palma, 2003).

En primer lugar, se presentan algunos hitos del proceso sociohistórico de la Mesa. En la trayectoria compartida "El camino" se reconstruyeron hitos significativos para las participantes desde su trayectoria objetiva y subjetiva. La aplicación de la técnica logró identificar principalmente los siguientes momentos:

1. La creación del proyecto como un desafío mayor de la Comunidad Económica Europea liderado por Greta Fajardo. Se logra visibilizar la temática a través del levantamiento de un diagnóstico de ESCNNA que se presenta en Seminario regional. Se decide conformar la organización.

2. Etapa de constitución de la Mesa con diferentes personas de distintos servicios del Estado, AURA y Universidad Católica de la Santísima Concepción (UCSC), marcada por tiempos variables y poca claridad de objetivos comunes.

3. Búsqueda de objetivos comunes y desarrollo de acciones de la Mesa en la sensibilización comunitaria y acercamiento a los procesos que afectan a los NNA. Se presentan conflictos con SENAME, se inicia proceso de "autonomía", incorporación de nuevos enfoques y desafíos.

Con diferentes perspectivas sobre su inicio, desarrollo y proceso de consolidación, las participantes refirieron:

Lo hice un poco sinuoso al principio (gráfico del camino) con estas subidas y bajadas y al final, yo creo que hay un tema de consolidación. (P1-técnica el camino)

Es un camino que se inició muy estrecho y que se va ampliando, y esta amplitud, tiene que ver no solo por el número de integrantes que se han ido sumando, sino también como la Mesa ha ido extendiendo su accionar. (P7-técnica el camino) 
Luego las participantes destacaron el proceso de administración inicial por parte de Servicio Nacional de Menores (SENAME), definido por las participantes como un "control SENAMISTA", causando un proceso rígido y prevalencia en paradigmas institucionales que desplazaban la participación y trabajo interdisciplinario por voluntades particulares:

Era un proyecto nacional, el proyecto no era de SENAME, aunque se veía así. (P7-técnica del camino)

El trabajo habría sido inicialmente dirigido con objetivos establecidos por el gobierno de turno, evaluados sobre la base del logro de metas cuantitativas, frente a lo que no necesariamente las integrantes de la Mesa estaban de acuerdo desde su mirada sociocrítica. Lo anterior fue lo que generó una separación paulatina entre SENAME y la Mesa Técnica. Las participantes se refirieron a una "independencia" en la toma de decisiones que favoreció una real participación y cierta autonomía, desplazando intereses particulares de por medio y restándose de algunos espacios de poder, pero a la vez permitiendo la generación de nuevas ideas de rápida incidencia y ejecución, sin largos procesos burocráticos que interfirieran al real sentido de la Mesa Técnica en la protección de los NNA.

A partir de 2015 y hasta 2016, la Mesa percibió avances sin mayores repercusiones o impactos en los diferentes espacios en los que se trabajaba, generando sensaciones de agotamiento y estancamiento. En 2017 se da un momento de reflexión en torno al sentido y al compromiso de cada uno de sus integrantes, concluyendo que la Mesa no es "técnica", sino "humana", significando con esto que logra cumplir con sus objetivos, porque cada una da valor al trabajo de sensibilización, visibilización, promoción, difusión, en su espacio de incidencia. Reconocen que su trabajo contra ESCNNA es necesario y urgente de realizar, "porque existen cada día más niños y niñas que están siendo gravemente vulnerados por este flagelo" (P2-técnica el camino).

Desde la técnica del flujograma, la Mesa identificó nudos críticos y aspectos en los que puede incidir o trabajar en mayor o menor 
Experiencia colaborativa de la Mesa Técnica contra la Explotación Sexual Comercial de la niñez en Bíobio / Gómez y Cid

medida, según la relación de poder con el problema, conflicto o necesidad. Las participantes realizan la siguiente reflexión: "a la sociedad no le importa si un niño es víctima, sino es más importante el daño que él o ella está causando, más que el daño que todos le hacemos, producto de la indiferencia" (P3).

Las entrevistas semiestructuradas realizadas a las participantes dieron como resultado elementos en común: las estrategias y las acciones parten de principios y valores básicos, y responden a una comprensión colectiva del fenómeno, desde las distintas miradas.

Primero todo el principio que se basan los derechos humanos, derechos humanos en general y los derechos de los niños y las niñas, también el principio de igualdad en procedimiento de justicia. (P7)

Así, también, identificaron la necesidad de desarrollar estrategias basadas en la evidencia, demostrando capacidad de cuestionamiento y creatividad.

Cuando conversamos, cuando elaboramos temas, escuchar las conversaciones siento que para mí también es gratificante estos aspectos, aunque el tema, eh... es un tema complejo, es gratifcante esto de compartir con las compañeras de la Mesa, elaborar algunos aspectos y conocer lo que ellas hacen, aprendo, cada día yo aprendo algo cuando voy. (P4-entrevista individual)

Reflexionaron sobre nuevos desafíos, principalmente el incorporar instituciones claves.

Estamos entrando a otra etapa, a la etapa que tenemos que ampliar esta mesa, porque si no, vamos a seguir dando vuelta sobre lo mismo. (P4-entrevista individual)

Lograron reconocer deficiencias en los procesos de seguimiento y evaluación de las acciones emprendidas.

Los resultados de los talleres, las evaluaciones de las capacitaciones, como las evidencias, pero no tenemos un trabajo sistematizado, no hemos logrado. (P7-entrevista individual) 
Este proceso de análisis dio inicio a la extracción de datos codificados en aquellos elementos técnicos clave para la interpretación del discurso obtenido de las protagonistas, unido a bases teóricas que fundamentan su relato y fiel exégesis según lo referido por ellas.

De esta forma, se pretendió ampliar la mirada del trabajo de la Mesa Técnica Regional contra la ESCNNA, buscando multiplicar las interrogantes, para analizar la continuidad (hacia dónde va), y mejorar su trabajo a partir de sus experiencias y aprendizajes. No solo se tuvo en cuenta el contexto en el que se dice y frente a quién se dice, sino el contexto temporal, es decir, ver qué sucedió antes y qué puede llegar a suceder (Schettini y Cortazzo, 2015). La lógica consistió en observar el proceso desde los marcos teóricos de la sistematización (enfoque constructivista, teoría de la complejidad y teoría fundamentada), para luego identificar aquellos elementos teóricos extraídos desde el discurso de las participantes en la integración de datos y enfoques teóricos, sin una contaminación teórica anticipada.

Se exploró el eje metodológico, constatando la convicción de las participantes que el problema debía ser abordado de manera integral, desde las voluntades institucionales y responsabilidad social frente a la protección de NNA en riesgo de ESC y/o sobrevivientes de dicha vulneración de derechos.

El fenómeno es un monstruo grande, digamos tan amplio, atraviesa todos los niveles sociales, todos los niveles culturales, atraviesa todas las manifestaciones. (P2-entrevista individual)

En el trabajo intersectorial dentro de su trayectoria, se reconoció periodos de responsabilidad y guía que asume SENAME en la gestión y ejecución de la Mesa, identificado por las participantes como un "proceso rígido" y estancamiento "senamista", provocando un quiebre y una paulatina separación con el servicio público.

La Mesa no era de SENAME y que teníamos objetivos propios, porque el SENAME quería que dieran cuenta a las metas que te- 
Experiencia colaborativa de la Mesa Técnica contra la Explotación Sexual Comercial de la niñez en Bíobio / Gómez y Cid

nían ellos como Estado, y nosotros como Mesa queríamos hacer otras cosas, distintas. (P7-técnica el camino)

Como análisis de este "proceso de independencias en la administración de SENAME”, se distinguió entre los beneficios y las desventajas que produjo, ya que, si bien se obtuvo mayor participación y libertad para la toma de decisiones, se perdió contacto con una institución crucial en la protección de los NNA. Esto, refirieron, por una falta de estrategias con aquellas instituciones que no adhieren a su formato de trabajo, que sin embargo son necesarias en materia de infancia, deformando su visión sistemática como práctica originaria.

Por lo tanto, para ellas, el primer punto a trabajar desde su metodología fue reconocer su trabajo sociocomunitario, desde lo complejo que conlleva trabajar con distintas organizaciones, intencionando la sensibilización sobre una problemática social poco visibilizada. De esta forma, las participantes definieron el trabajo en ESCNNA, integrando en su narrativa elementos del paradigma de la complejidad, de la mirada sociocrítica y también desde el enfoque humanista; reconociendo este último como esencia en la creación y mantención de la Mesa, siendo un aspecto importante desde su identidad y compromiso social de las participantes.

Tenemos que abrirnos a conocer otras gentes, que también va a ser positivo pero que también a mí me da un poquito de susto en ese aspecto, pero también creo que es importante enfrentar esta situación. (P4-entrevista)

Por otra parte, según el eje ontológico, las participantes agruparon los problemas, necesidades insatisfechas y conflictos, su implicancia en la generación de conocimientos y discursos, reconociendo un alto poder sobre estos.

Dimensionaron un proceso sistemático y circunstancial desde sus causas-efectos, logrando codificarlos dentro de tres categorías: bloques estructurales de resistencia; condiciones metodológicas, y alianzas estratégicas. Se reforzó la idea de que los nudos 
reconocidos son causales de una estructura mayor de dominación desde dimensiones culturales, sociales, económicas y políticas, tal como lo refirieron al señalar: el "Sistema emergente, referido a una economía neoliberal centrada en la producción y la ganancia favoreciendo la cosificación y compraventa de los cuerpos de los NNA", puesto que al comprender este elemento macro de incidencia sobre los demás, proporciona una mirada holística en el abordaje de ESCNNA, pero también de violencia estructural y simbólica.

Lo anterior, se relaciona con la investigación de Medina sobre ESCNNA, que refiere aquellos elementos estructurales que influyen en el abordaje, señalando que éste no es un fenómeno nuevo, extraño, ni ajeno; por el contrario, es un problema estrechamente vinculado a la pobreza y a la marginalización, pero también íntimamente relacionado con todas esas ideas, mitos y prejuicios con las que los y las guatemaltecas constituimos nuestras relaciones de género y generacionales. (Medina, 2016)

Me refiero a justicia en su aspecto más amplio, porque esta es una situación de injusticia atroz, que atraviesa y transforma negativamente el futuro... es una cuestión que debe ser erradicada, entonces... ¿por qué?, porque es injusto. (P2-entrevista individual)

Las participantes de la Mesa analizaron la necesidad de una intervención sociocomunitaria que logre sensibilizar a la población, permitiendo ver el fenómeno como algo cercano, asumiendo una responsabilidad como garantes corresponsables en defensa de los NNA.

Con relación al poder o subordinación de la Mesa sobre los problemas metodológicos del abordaje de la temática, se reconoció un alto poder de incidencia sobre ellos, por medio de gestiones resultado de la experiencia y la vinculación de actores clave, aunque a veces no tuvieron una respuesta favorable o les fue indiferente. No obstante, destacaron los esfuerzos y avances que ya habían logrado, aun con la falta de apoyos institucionales y recursos, 
trabajando desde las voluntades personales y compromisos de las participantes; concluyeron que, si bien es viable y positiva esta independencia y flexibilidad institucional, existe una deficiencia en la real responsabilización por parte de las instituciones, asumiendo una labor de respaldo social como razón y nombre reconocido socialmente. Declararon que en aspectos prácticos no existió un marco que estableciera claramente los compromisos por parte de la organización y que permitiera desde un comienzo establecer recursos, designación de tiempos, entre otros.

De esta forma, la Mesa asumió los nuevos desafíos con un alto poder en su dominio, aun cuando reconocieron que persisten barreras estructurales que complejizan la intervención.

Reconocieron que se precisa la reconceptualización técnica, un saber propio, que refuerce su trabajo, con iniciativas en diferentes niveles sociopolíticos y en la incorporación de capital social comunitario.

Concluyeron que los desafíos y dificultades son constantes, pero que, al ser identificadas de manera correcta con un real sentido para la protección de los NNA, se trasforman en una cadena de hechos que favorece un replanteamiento en las intervenciones, permitiendo una adecuada anticipación a sucesos de vulneración, logrando el significado de la prevención: generar condiciones para el ejercicio real de los derechos humanos con las garantías del Estado.

En el eje epistemológico, se analizó el saber, se realizó un estudio del conocimiento producido. Declararon al respecto que se vive una fuerte "crisis de subjetividad positivista moderna", pero que ellas han insistido, desde las ciencias sociales, en comprender la realidad como construida y capaz de reconstruirse socialmente. El poder trabajar en terreno, in situ, permitió a la Mesa recopilar nuevo conocimiento, a través de la conceptualización de algunos términos y constructos.

Carvajal (2010), en su investigación sobre desafíos para la educación superior y la investigación, señala que existe una necesi- 
dad de implementar una visión holística, integral e interdisciplinaria para resolver los problemas actuales, que solo se logra con el trabajo en equipo, señalando diferentes conceptos de abordaje que reconstruye su experiencia profesional en equipos interdisciplinarios, multidisciplinarios y transdisciplinarios. Aseguraron las participantes una diversidad de conocimiento y visiones de la realidad dentro de la Mesa, que varían desde sus instituciones, profesiones y personalidades, por lo que generar una visión común presentó un gran desafío, que sortearon definiendo objetivos comunes. También les ayudó la dinámica de relación horizontal, sin una asignación de cargos o jerarquización, que potenció el trabajo colectivo y emancipador. Sin embargo, habrían persistido rincones de pasividad en los que la tarea fue focalizada a una sola área, lo que trunca la potencialidad en el trabajo unificado.

El grupo es muy agradable, yo lo paso muy bien cuando conversamos, cuando elaboramos temas, escuchar las conversaciones, siento que para mí también es gratificante en ese aspecto, aunque es un tema complejo, es edificante esto de compartir con las compañeras de la Mesa, elaborar algunos aspectos y conocer lo que ellas hacen, aprendo, cada día yo aprendo algo cuando voy. (P4-entrevista individual)

En relación con cómo las participantes reconocieron su trabajo, desde la creación de conocimiento y selección de estrategias en diversas actividades, ellas apreciaron el apoyo y trabajo de conexión interinstitucional, como indicador de avance en la protección de NNA.

Estoy pensando cómo ha sido el aporte directamente a los niños y niñas, en particular los vínculos con la Fiscalía, siento yo, esto de "oye ...mándame un correo", para mí eso es como súper significativo, y con la Católica, esto de aportar en capacitación en la conversa en el enfoque en la mirada, para mi por lo menos ha sido un aporte directo para el proyecto, que yo sé que ha sido un vínculo de confianza. (P3-técnica el camino) 
Asimismo, el trabajo se posicionó desde un problema complejo, lo contrastaron con la acción institucional, que ha abordado históricamente una focalización simple, como cualquier programa social, y que no habría generado resultados deseados.

Uno igual se da cuenta de que la academia, aunque sea de Trabajo Social, no investigación, no está hecha para eso, no está hecha para las personas, está hecha para publicaciones, o para figurar en datos estadísticos. (P6-técnica el camino)

Entonces hay una barrera, yo siento que he fracasado profundamente, yo no he sido un aporte para esto, de ninguna manera... a lo mejor yo tengo la idea que me tengo que sumar a lo que ustedes tienen, y yo no encuentro mi nicho aquí, y que donde yo veo mi nicho, no encuentro la recepción, entonces tengo un sentimiento de profunda frustración. (P5-técnica el camino)

Reconocieron un trabajo interdisciplinario, avanzando hacia la transdisciplinariedad, argumentaron que, en sus reuniones técnicas de análisis o planificación, cada una de las participantes aporta desde sus disciplinas e instituciones.

Expresaron orgullo por estar más allá de sus disciplinas, abriendo espacios a nuevos conocimientos, con una riqueza epistemológica que nació desde sus propias experiencias.

La autorreflexión del proceso de sistematización fue coherente con sus conclusiones, entre las que destacó la riqueza que cada una ha representado como baluarte único en el posicionamiento frente a ESCNNA, pero, a la vez, con un fiel discurso colectivo en contra de toda manifestación que vulnere la dignidad y derechos de los niños, niñas y adolescentes.

Igualmente, destacaron que su trabajo generó una atmósfera de valor humano, desde el respeto por la opción del otro, en la que interactúan distintos pensamientos, profesiones, principios, valores, paradigmas, etc. Un espacio de contención emocional y autocuidado para quienes de desempeñan laboralmente en programas 
de reparación del daño, que sin duda enriquecen la labor, aunque complejice la tarea al momento de buscar consensos. Se aprendió a dejar y ceder en ocasiones, por el alcance global en la protección de los niños, niñas y adolescentes.

\section{Conclusiones}

Según la CEPAL-ONU, los problemas modernos deben estudiarse desde varias disciplinas y conformando equipos, para obtener soluciones integrales, dado que ninguna disciplina, por separado, puede dar resultados por sí misma (Carvajal, 2010). Los equipos, como la Mesa, pueden aportar muy poco si contribuyen únicamente con una visión técnica, sin integrar su conocimiento con las demás disciplinas.

La sistematización demuestra que la incorporación de actores en la prevención y enfrentamiento de la ESCNNA representa una estrategia efectiva, señalando que cuantas más personas se hacen responsables ante la tarea de hacerle frente, mayores son las posibilidades de que se logre detectar, proteger a las víctimas, sancionar a los explotadores y finalmente prevenir que ocurra (Araya, Retuerto y Tarducci, 2012). El abordaje de la Mesa ante este fenómeno comprende acciones en los diferentes sistemas sociales y estructurales, concientizando y apuntando a un cambio micro como macrosocial para la comprensión y sensibilización sobre ESCNNA.

La Mesa Técnica contra la ESCNNA ha venido asumiendo el reto que tiene el Estado, la sociedad civil y la academia, de accionar en dicha problemática compleja. Esto le ha permitido reconocer aspectos en los que debe exigir la acción gubernamental como garante de los derechos humanos de todos los niños, niñas y adolescentes originarios y que residan en nuestro país. Es claro que la responsabilidad en la garantía de los derechos humanos descansa en el Estado, por esto la Mesa es consciente de su alcance real, siendo crucial el trabajo de incidencia en aspectos de mejorar las políticas públicas dirigidas a ESCNNA como una estrategia a lar- 
go plazo, de mucha especialización, que integre a diversos dispositivos para distintos territorios, modalidades y etapas de la vida de las víctimas, impulsar la Ley de Protección Integral de la Infancia, Defensoría de la Niñez en la región, actualización de la legislación penal, generación de equipos investigativos y policiales especializados, y otras medidas para un trabajo en conjunto que no quite responsabilidad al Estado.

Lo anterior, se refuerza con los resultados de la investigación señalada ${ }^{8}$, que explica que es imprescindible el fortalecimiento de la atención en salud mental para las víctimas, portadoras de trauma complejo, en casos en que la explotación sexual es una finalidad de la trata de personas, ya sea en su modalidad trata interna, transitoria o autogestionada (ONG Raíces, s/f), sus efectos en los NNA serían similares a los de la tortura (CEDOC, 2010).

Se requiere una nueva institucionalidad para la niñez, una política amplia e integral, que se sustente sobre una oferta programática permanente, pertinente, situada, financiada bajo el amparo de la protección integral de los derechos humanos y el acceso a la reparación del daño en dispositivos interdisciplinarios y mantenidos en el tiempo. No puede continuar la lógica de mercado, de competencia de proyectos cada dos años a través de licitaciones para responder a las víctimas, es reparación del daño causado a las personas más vulnerables de la sociedad, polivictimizadas: la reparación y resignificación de la experiencia vivida es también un derecho a ejercer.

El trabajo de la Mesa constituye un avance significativo en la prevención, detención, capacitación, intervención y protección de los NNA que pudieran estar en riesgo o expresamente como víctimas de ESC. Ha logrado permanecer en el tiempo, aun con dificultades, estableciendo instancias reconocidas y esperadas por el intersector, sus seminarios, cuentas públicas, sesiones de análisis

Ortega, M., Gómez. V (2015). Resultados Proyecto DIN 'Trayectoria sentidos y sentimientos". 
de casos, talleres abiertos, producción de gran cantidad de material gráfico, cartillas, volantes, dípticos, revistas, etc. Además de su permanente presencia en redes sociales, orientaciones por medio de correo electrónico ${ }^{9}$ y noticias de su página web $^{10}$, sintiéndose validada por su medio social por su reconocida responsabilidad en la temática. No obstante, advierten sus miembros, la validación no ha logrado producir los apoyos logísticos y materiales necesarios para el desarrollo de la organización, que persiste aun cuando puedan variar sus integrantes. Ellos se plantean formalizar la organización a través de registros públicos, cartas de membresía, presupuestos, convenios, entre otros, para no desviar la energía de las participantes en estos temas y favorecer una mejor práctica de intervención.

De igual forma, destacan la importancia de contar con representatividad en aquellas instituciones en las que la toma de decisiones en materia de infancia es crucial, tal como el caso de SENAME, que, si bien la separación demuestra un hecho favorable en el trabajo de la Mesa, se distancia un ente decisivo en ámbitos de incidencia política. Los desafíos siguen siendo mayores con las nuevas figuras en materia de infancia, como la Defensoría de la Niñez, y el posicionamiento frente a la fuerte crisis que atraviesa SENAME. La Mesa desea definir su adherencia y opinión sobre la problemática, siendo reconocida por todas sus integrantes como parte de su constructo operacionalista.

La necesidad de nuevos actores integrantes planteada por la Mesa se visualiza como una selección estratégica. Se propone identificar aquellos que pueden ser "capital social escalera", aquellas organizaciones o personas que se igualan en los objetivos que tiene la Mesa o en su trabajo directamente con la población en cuestión, fundamentalmente las policías, Tribunales de Familia y equipos de salud integral, y también prestar atención al "capital puente", referido a aquellas organizaciones y/o personas que, sin tener co-

mesaescnna@gmail.com

http://www.mesaescnnabiobio.com 
nexión con los objetivos de la Mesa, poseen una riqueza en la vinculación con aquellos que generan mayor resistencia, avanzando en el abordaje y participación comunitaria como movilización del tejido social. Es importante involucrar a los sectores del comercio, turismo, municipalidades, y de acuerdo con los actuales flujos migratorios, a extranjería y representantes del Ministerio de Desarrollo Social y de la Mujer y Equidad de Género.

La Mesa espera incluir la participación de los y las protagonistas NNA y sus familias o cuidadores, sus experiencias y recomendaciones; sus historias y situación actual, identificando aquellos elementos que favorecieron la resignificación del daño y los que dificultaron su proceso. Esto responde al interés de las integrantes de la Mesa Técnica como actoras que vivencian la unidad entre sujeto y objeto del conocimiento (Ghiso, 2008), para la comprensión, reflexión y aplicación de nuevas estrategias, con especial énfasis en la participación y validación de las trayectorias y aprendizajes desde la voz de los sobrevivientes de ESCNNA. Además, con ello lograrían flexibilizar el formato un poco autorreferente y profesionalizante de la Mesa, para conformar consejos más comunitarios. Con nuevas alianzas estratégicas, no solo se puede fortalecer la acción de la Mesa, sino también resolver aquellos nudos críticos que persisten. Si bien está suficientemente consolidada y goza de cohesión interna, abrirse a nuevos participantes no representa una amenaza; por el contrario, rigidizar sus límites podría significar un retroceso, como da cuenta la experiencia narrada.

Desde el eje metodológico, declararon fundamental revisar su propósito o misión, y planificar estratégicamente sus acciones, siempre con interdependencia del contexto histórico-social y político. El por qué y cómo, lo consideran definido, pero no declarado. Identificaron como necesidad, normas de autorregulación, estrategias táctico-operacionales, como directrices que guíen una planificación a largo plazo, con indicadores específicos para cada acción, siempre desde un análisis constante y vigilancia de la problemática y su dinamismo. Tal análisis nunca será objetivo, por su carácter social y humano; sin embargo, las ciencias sociales deben 
ser capaces de anteponerse y analizar aquellos hechos sociales que logren mitigar los efectos y daños a las personas (Huerta, 1994). La planificación estratégica busca más resultados que generación de actividades orientadas a generar decisiones fundamentadas, fijar metas y desarrollar los instrumentos para lograrlo. La Mesa planteó hacia dónde quiere llegar, pero debe construir los caminos posibles. Declarar en su propuesta los marcos teóricos y normativos que orientan la acción, que ya están consensuados, también es muy importante si la Mesa se abre a nuevos actores.

La debilidad metodológica más importante de algunos proyectos consiste en su incapacidad para documentar los objetivos programáticos próximos (Cantón y Cortes, 1998). Un plan de acción bianual de La Mesa, que coincida con la rotación de la coordinación, con objetivos específicos, metas, indicadores, medios de verificación, actores, además de fundamentación, valores, referentes teóricos, constructos, valores éticos, adherencia política, que permita traspasar el conocimiento colectivo a un producto a disposición de los demás. De igual forma, les resulta importante establecer mecanismos de evaluación de resultados y del impacto que el trabajo de la Mesa pudiera tener en incidencia política, protección de las víctimas, denuncias y formación especializada de pre y posgrado.

Acompañar esta vulneración extrema de NNA, compleja, dinámica, marcada por procesos sociohistóricos, les significa comprender integral e integradamente, multidimensional y convergentemente, trascender polarizaciones y descalificaciones acrisoladas desde la modernidad- racionalidad sobre las relaciones, conocimiento, saber, poder, cambio social, construir vínculos entre lo estructural y coyuntural. Precisar relaciones tríadicas, inclusión de conceptos, procesos complejos (Brand Barajas, 2016)SIN REFERENCIAS.

Se requiere evaluar si las acciones de la Mesa responden a la complejidad y magnitud del problema, si pueden atravesar la esfera del medio próximo y avanzar en aquellos que presentan mayor resis- 
tencia, como turismo, salud, instituciones de orden público, vendedores ambulantes, comunidad en general. Es un desafío mayor para la articulación Estado-sociedad civil organizada y academia presente en la Mesa. La promoción de la movilización y la denuncia en el tejido social; ser agente de prevención y denuncia en entornos cercanos, como la familia, el barrio y los lugares de trabajo; desde allí, los asuntos se vuelven públicos, comunes, de interés colectivo y exigen la participación de todas las personas (Fundación Renacer, 2011, p. 68).

Esta sistematización dio cuenta de aprendizajes obtenidos en la trayectoria de La Mesa; sin embargo, no existe claridad acerca de cómo validarla externamente, ni de cómo avanzar en la integración coherente de las intencionalidades ideológicas y políticas de las distintas instituciones participantes con opciones epistemológicas. La Mesa se debe instituir y legitimar como organización experta en la temática, para asesoramiento, formación e incidencia en política pública. Este modelo de trabajo puede ser un real aporte a miles de mesas temáticas en Chile, orientadas a la transformación del tejido social. Se está en condiciones de generar un modelo de atención integral sobre ESCNNA, que logre ampliar la mirada de la intervención no solo en la víctima y su entorno más próximo, sino a un enfoque comunitario, con elementos culturales pertinentes. Luego de esto, es esencial poder socializarlo, por medio de un intercambio de experiencias, que pudiese lograr la replicación en otras regiones y espacios.

La Mesa siente el desafío del cambio sociocultural que se está viviendo en el aumento de personas extranjeras en situación de migración forzada por razones de gran dificultad en sus países de origen, especial preocupación por los NNA que puedan ser captados, engañados e involucrados en explotación sexual.

De acuerdo con la Organización Mundial del Turismo (OMT), existen viajes organizados desde dentro del sector turístico, o desde fuera del sector, pero utilizando sus estructuras y redes, con el objetivo de que el turista tenga relaciones sexuales con ni- 
ños, niñas y adolescentes residentes en el destino (ONG Raíces, s/f, p.3).

La Mesa se reconoce trabajando en establecer y difundir líneas estratégicas desde la ética y responsabilidad social sustentable, tratando de actuar a tiempo para disminuir las posibilidades de vulneración sobre la población emergente por medio de iniciativas interinstitucionales, pero las barreras burocráticas, tanto al interior de sus propias instituciones como fuera de ellas son importantes; plantean que han logrado avances desde aquellas instituciones menores, como el comercio ambulante, hostales, etc., que permiten fortalecer la prevención en los espacios barriales y cotidianos.

La Mesa Técnica contra la ESCNNA se auto refiere como especializada en la temática, marcada por las características de sus participantes, diversa pero no dispersa, su trayectoria de construcción colectiva ha mostrado variadas intensidades y momentos, se ha logrado diferenciar del Estado para enfrentar y subsistir ante cambios en la política pública de niñez de mayor o menor subordinación, y a procesos sociohistóricos que afectan directamente a su grupo prioritario. Su experiencia y formación en la temática es riquísima, tiene mucha producción de material académico y socioeducativo, y ha logrado mantener relaciones sociales colaborativas con participantes y sujetos de atención de los programas de manera transversal y a través del tiempo.

Los desafíos que se plantea van por la validación y apoyo institucional, del cual no reconoce resistencia, pero tampoco mayor solidaridad. Esto debilita su capacidad de acción en cuanto a recursos y tiempos disponibles. La coordinación rotativa ha sido un factor facilitador. Sus integrantes están conscientes de la necesidad de formalización, apertura y crecimiento, y tienen todo lo necesario para avanzar en ese camino. 
Experiencia colaborativa de la Mesa Técnica contra la Explotación Sexual Comercial de la niñez en Bíobio / Gómez y Cid

\section{Bibliografía}

Araya, D., Retuerto, I. y Tarducci, G. (2012). Comunidades sensibilizadas y alerta a la Explotación Sexual Comercial de Niños, Niñas y Adolescentes. Santiago, Chile: ONG Raíces, Fundación San Carlos.

Barnechea, M. \& Morgan, M. (2007). El conocimiento desde la práctica y una propuesta de método de sistematización de experiencias. Trabajo para optar al grado de Magíster en Sociología, Lima, Pontificia Universidad Católica del Perú.

Barudy, J. y Dantagnan, M. (2011) La fiesta mágica y realista de la resiliencia infantil. Gedisa Editorial.

Brand Barajas, J. (2016). Tríadas conceptuales / tríadas del saber. Experiencia docente universitaria. I Simposio internacional y $\mathrm{V}$ institucional de experiencias docentes universitarias. Bogotá: Universidad de la Salle.

Cantón, J. y Cortés, M. (1998). Malos tratos y abusos sexuales infantiles. España: Siglo Veintiuno de España Editores S.A.

Carvajal, Y. (2010). Interdisciplinariedad: desafío para la educación superior y la investigación. Revista Luna Azul ISSN 19092474. Manizales.

CEDOC (2010). Diagnóstico sobre el Fenómeno de Trata de Personas para determinar su impacto en mujeres y niñas en el Estado de Oaxaca. Ediciones Instituto de la mujer Oaxaqueña.

Cifuentes, R. (1999). La sistematización de la práctica en Trabajo Social. Argentina: Editorial Luman Humanitas. Colección procesos y políticas sociales.

Cifuentes, R. (2015). La sistematización en Trabajo Social: entre la práctica y la investigación, en Reflexionando las disciplinas (pp. 129-160). Universidad Mariana.

ECPAT. (2014). Informe de Monitoreo de país sobre la Explotación Sexual Comercial de Niños, Niñas y Adolescentes. Thailand: ECPAT Internacional.

Fundación Renacer. (2011). Sistematización del Proyecto para Prevenir la Explotación Sexual Comercial de Niños, Niñas y Adolescentes en el contexto de viajes y turismo en la ciudad de Cartagena. Recuperado de https://fundacionrenacer. org/wp-content/uploads/2016/03/sistematizacion-final5-dic-2011.pdf

Ghiso, A. (2008). La sistematización en contextos formativos universitarios. Medellín Colombia: Fundación Luis Amigó. Recuperado de http://www.alforja.or.cr/sistem/sistem_old/Articulo_Funlam.pdf 
Huerta, F. (1994). Entrevista a Carlos Matus. Recuperado de https:// www.cepal.org/ilpes/noticias/paginas/2/36342/libro_entrevista_con_matus.pdf

Jara, O. (2012). Sistematización de experiencias. Práctica y teoría para otros mundos posibles. San José: Intermón.

Jiménez R., García, E., Azcárate, P., Navarrete, A. y Cardeñoso, J. (2016). La Teoría Fundamentada como estrategia de análisis de los datos: caracterización del proceso. Revista de Investigación Cualitativa en Educación, I(1), 356-355.

Kvale, S. (2011). Las entrevistas en investigación cualitativa. Madrid: Morata.

Lindón, A. (2007). El constructivismo geográfico y las aproximaciones cualitativas. Revista de geografía Norte Grande, (37), 5-21. DOI: https://dx.doi.org/10.4067/S071834022007000100001

Londoño, D. \& Atehortúa, G. (2011). Los pasos en el camino de la sistematización. Decisio, 28, 30-34.

Luengo, E. (2008) La simplicidad del método científico y la complejidad de lo real. Recuperado de https://rei.iteso.mx/bitstream/ handle/11117/1040/simplicidad\%20m\%C3\%A9todo\%20 y\%20complejidad $\% 20 \% 282 \% 29$.pdf? sequence $=2$

Medina, M. (2016) Sistematización de las Redes contra la Explotación Sexual Comercial, la importancia de la articulación. Experiencia en Guatemala. Instituto interamericano del niño, la niña y adolescente.

Ministerio de Justicia y Derechos Humanos de Chile, (2017). Tercer Marco para la Acción contra la Explotación Sexual Comercial Infantil y Adolescente. Secretaría ejecutiva. Recuperado de http://www.minjusticia.gob.cl/media/2018/01/3erMarcodocumento2017-2019.pdf

Morin, E. (2011). Introducción al pensamiento complejo. Barcelona: Editorial Gedisa.

Movimiento el Pozo. (2015). ¿Por qué tenía que sentir miedo, si ahora tengo otra vida? Reflexiones y aprendizajes sobre atención integral y empoderamiento en mujeres vinculadas al comercio sexual. Lima, Perú: Movimiento el pozo.

ONG Raíces. (s/f). Material didáctico para la prevención, detección temprana y protección de niños, niñas y adolescentes víctimas de explotación sexual comercial. Explotación Sexual Comercial Niños, Niñas y Adolescentes (ESCNNA). Recuperado de https://www.ongraices.org/admin/doctos/doc_6. pdf 
Experiencia colaborativa de la Mesa Técnica contra la Explotación Sexual Comercial de la niñez en Bíobio / Gómez y Cid

ONG Raíces (2017). Caracterización de las formas de explotación sexual comercial de niños, niñas y adolescentes. Consejo nacional de la infancia. http://observatorioninez.consejoinfancia.gob.cl/publicaciones/estudio-caracterizacion-de-lasformas-de-explotacion-sexual-comercial-de-ninos-ninas-y-adolescentes.

Palma, D. (2003). La sistematización como validación de la intervención social. Encuentro de experiencias de intervención en el ámbito familiar y comunitario, Concepción: Universidad de los Lagos.

Pérez. T. (2005). La perspectiva constructivista en la investigación social. Revista Tendencia y Retos, 10, 39-64. Recuperado de http://www.ts.ucr.ac.cr/binarios/revistas/co/rev-co-tendencias-0010-03.pdf.

Rivera, F. (2011). Argumentación y construcción de validez en la Planificación Estratégica-Situacional de Matus. Cuadernos de Saúde Pública, 27(9), 1847-1858. DOI: https://dx.doi. org/10.1590/S0102-311X2011000900018

Schettini, P. y Cortazzo, I. (s/f). Análisis de datos cualitativos en la investigación social. Argentina: Universidad Nacional de la Plata.

Servicio Nacional de Menores. (2015). Programas especializados en ESCNNA y Cobertura. Recuperado de http://www.sename.cl/ wsename/otros/DOCPFTI/30-01-2015/OFERTA-ESCNNA.pdf.

Servicio Nacional de Menores. (2016). Observatorio Abuso Sexual Infantil y Adolescente. Chile: Ministerio de Justicia. Recuperado de http://ddhh.minjusticia.gob.cl/media/2016/12/informe_sename_digital_116_pagabuso_sexual_en_chilepdf. pdf. 